\title{
Peripheral artery disease and unusual skin findings
}

\begin{abstract}
A 68-YEAR-OLD MAN with peripheral artery disease and dry gangrene of the feet was admitted to the hospital from a subacute rehabilitation facility because of increasing edema and erythema in the right lower extremity, with scattered areas leaking serous fluid.

Five months previously, he had presented to a podiatrist with superficial ulceration of the fifth toe of his left foot and a purple hue to all toes of both feet, findings that were thought to represent ischemia. Evaluation for vasculitis indicated normal renal function and was negative for antinuclear antibody, rheumatoid factor, and cryoglobulins. His erythrocyte sedimentation rate was mildly elevated at $12 \mathrm{~mm} /$ hour (Table 1). Peripheral artery disease was diagnosed, manifesting as dry gangrene of his toes and feet. Risk factors for peripheral artery disease were treated optimally with antihypertensive drugs, a statin, and low-dose daily aspirin, although he continued to smoke.

Three months before the current admission, he underwent left femoral endarterectomy with angioplasty with the goal of treating his peripheral artery disease. However, the ischemic changes of his feet continued to progress, and he developed necrotic areas on the right foot (hallux and the fourth and fifth digits) and the left foot (hallux and the distal aspects of the second, third, and fifth digits). Three weeks before the current admission, he underwent angioplasty of the right superficial femoral artery.

At the rehabilitation facility, he received appropriate wound care for his gangrenous toes, but because of the severity he could no longer bear weight and required a mechanical lift for transfers. He had chronic lower doi:10.3949/ccjm.87a.19074
\end{abstract}

TABLE 1

The patient's laboratory evaluation 5 months earlier

\begin{tabular}{lll} 
Test & Value $^{\mathrm{a}}$ & $\begin{array}{l}\text { Reference } \\
\text { range }\end{array}$ \\
\hline Erythrocyte sedimentation rate & $\mathbf{1 2 ~ \mathbf { ~ m m } / \text { hour }}$ & $0-10$ \\
$\begin{array}{l}\text { C-reactive protein } \\
\text { Antineutrophil cytoplasmic anti- }\end{array}$ & $0.90 \mathrm{mg} / \mathrm{L}$ & $<5.00$ \\
bodies immunoglobulin (Ig) G & & $<1: 20$ \\
Rheumatoid factor & $10.2 \mathrm{IU} / \mathrm{mL}$ & $0-14.0$ \\
Cardiolipin IgA & $4.9 \mathrm{APL}$ units & $0.0-13.9$ \\
Cardiolipin IgG & $4.5 \mathrm{GPL}$ units & $0.0-9.9$ \\
Cardiolipin IgM & $<2.5 \mathrm{MPL}$ units & $0.0-9.9$ \\
Nuclear antibody IgG & $<1: 40$ & $<1: 40$ \\
Cryocrit & $<1 \%$ & $<1 \%$ \\
\hline
\end{tabular}

abnormal results are shown in bold.

extremity pain with no report of sensory loss. Lower extremity strength was 3 on a scale of 5 and symmetric bilaterally.

The patient's history also included tobacco use, hypertension, chronic obstructive pulmonary disease, hypothyroidism, urinary retention, and constipation. He had previously received the diagnosis of iron deficiency anemia, but iron supplementation had been recently discontinued because his hemoglobin level had returned to normal. His medications on admission to the hospital included lowdose aspirin, atorvastatin, carvedilol, lisinopril, levothyroxine, acetaminophen, senna, polyethylene glycol, albuterol and mometasone-formoterol inhalers, as well as albuterolipratropium given by nebulization. 


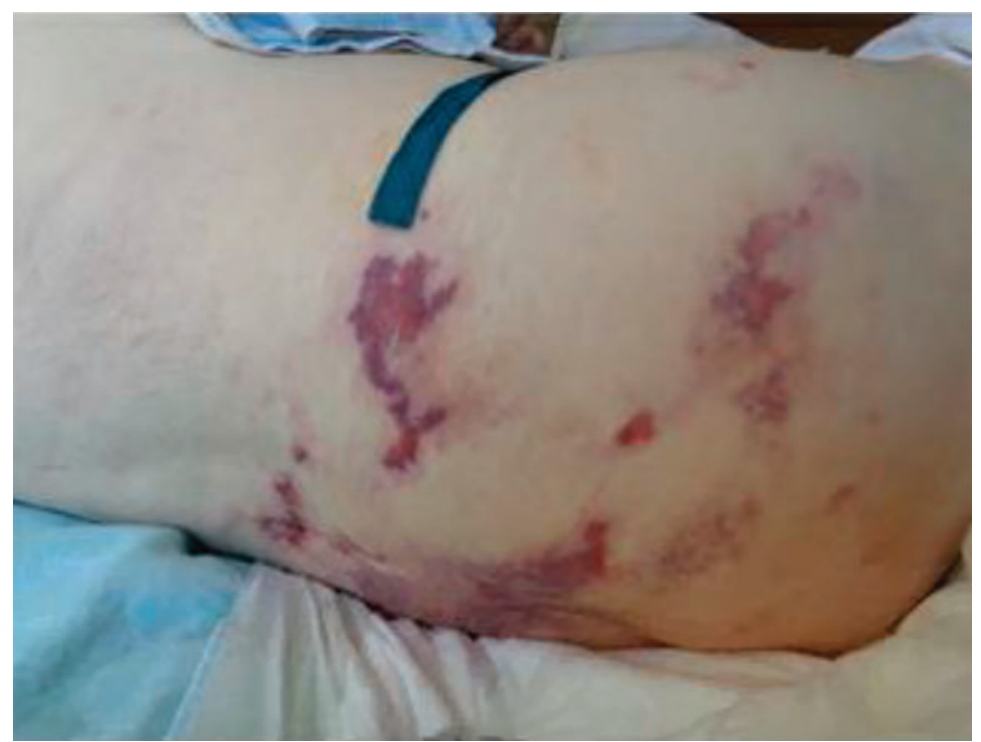

Figure 1. The patient's lower back and buttocks on initial presentation.

\section{A 68-year-old man was admitted because of worsening skin problems in his legs}

\section{Physical examination}

On admission, his blood pressure was 109/64 $\mathrm{mm} \mathrm{Hg}$, pulse 83 beats per minute, temperature $36.6^{\circ} \mathrm{C}\left(97.9^{\circ} \mathrm{F}\right)$, weight $84.4 \mathrm{~kg}$, and oxygen saturation $97 \%$ on room air. He was alert and in no distress.

Cardiopulmonary and abdominal examinations were unremarkable. His right leg had brawny edema with ill-defined reddish discoloration from the knee downward, with scant yellowish fluid weeping through small open erosions of the skin. The left calf appeared normal without edema. He had no lymphadenopathy.

He had a purplish red reticular discoloration of the skin around the right knee and diffuse red, purple, and maroon discolorations over the lower back and buttocks (Figure 1), known to be present for about 2 weeks. The skin appeared intact with blanchable discoloration.

The chronic necrotic changes of the toes appeared unchanged and without subungual hemorrhage. Posterior tibial pulses were difficult to palpate.

\section{Laboratory results}

A complete blood count with differential and a basic metabolic panel were drawn (Table 2). His thyroid-stimulating hormone level, liver enzyme levels, and international normalized ratio were normal.

\section{CHARACTERIZING THE SKIN PROBLEM}

Which of the following is most consistent with the patient's skin findings?

\section{Pressure injury \\ Ecchymosis \\ Livedo reticularis \\ Purpura fulminans}

Livedo reticularis is the condition most consistent with our patient's skin findings. A descriptive term indicating reddish-blue spots in a netlike or lacy pattern, livedo reticularis is caused by decreased blood flow to the skin.

The cutaneous vascular microanatomy can be thought of as a deep arterial bed with superficially penetrating arterioles for blood supply and a dense superficial venous plexus. Under this model, any process that reduces cutaneous blood flow results in better visualization of the venous plexus.

Physiologic vasospasm (eg, from cold exposure) can lead to reversible cutaneous discoloration, known as physiologic (primary) livedo reticularis.

Irreversible (secondary) livedo reticularis can lead to necrotic skin lesions owing to a lack of blood flow in the superficial arterial vessels. ${ }^{1,2}$ It has systemic associations and can be caused by the following:

- Hypercoagulable states such as antiphospholipid syndrome and thrombotic thrombocytopenic purpura

- Other autoimmune conditions, including various manifestations of vasculitis

- Conditions of embolization or deposition, such as cholesterol embolization syndrome and calcific uremic arteriopathy.

Pressure injury. The skin remains intact only in the first stage of pressure injury and deep-tissue pressure injury, in which the skin is ischemic but not infarcted. Pressure injury is most commonly diagnosed visually.

Unlike the findings in our patient, stage 1 pressure injuries are erythematous rather than purple-maroon and are typically described as nonblanchable. Although a blanch response may be elicited in early stage 1 pressure injuries, our patient's skin lesions were present in some form over the previous 2 weeks. Deep-tissue pressure injury is maroon or purplish in appearance but is also typically nonblanchable. ${ }^{3}$ 
In addition to these differences, the sites involved in this patient do not suggest pressure injury, making this diagnosis unlikely. Pressure injury typically involves skin over a bony prominence, such as the iliac crest, sacrum, or coccyx. ${ }^{4}$ Furthermore, although this patient is chair-bound, he is alert, has intact sensation, and is unlikely to have sustained pressure over the areas of these lesions to create a pressure injury.

Ecchymosis is the result of extravasation of blood in the tissues, most commonly from blunt-force trauma. Less commonly, it is seen with severe pancreatitis or peritoneal hemorrhage, ${ }^{5}$ with classic distributions being periumbilical (Cullen sign) and flank (Turner sign). ${ }^{6}$

Ecchymosis is unlikely in our patient, who has no history of trauma and no notable abdominal symptoms to suggest severe acute pancreatitis or retroperitoneal hemorrhage.

Purpura fulminans, despite its name describing deep purple lesions, is not related to ecchymosis. Purpura fulminans involves extensive infarction of the skin, with the petechiae and purpura quickly progressing to blood pooling and skin necrosis. Purpura fulminans can follow disseminated intravascular coagulopathy (intravascular thrombosis and hemorrhage) caused by bacterial infection, malignancy, or immunologic reactions. $\mathrm{Pa}$ tients are acutely ill with rapid deterioration; hence, purpura fulminans signals a dermatologic emergency. ${ }^{7,8}$

Although our patient's skin lesions were initially violaceous and purpuric, they progressed slowly and he was not acutely ill, so this diagnosis does not fit.

\section{CASE CONTINUED:} THE PROBLEM SPREADS

The patient's right leg erythema was thought to represent deep vein thrombosis, reperfusion injury from his prior angioplasty with reactive erythema and edema, or ischemic changes from progression of his peripheral arterial disease. Venous ultrasonography showed no evidence of deep vein thrombosis. Angiography of the right lower extremity was repeated, and balloon angioplasty of a newly found narrowing in the right femoral artery was performed.
TABLE 2

The patient's laboratory results on admission

\begin{tabular}{|c|c|c|}
\hline Test & Value $^{a}$ & $\begin{array}{l}\text { Reference } \\
\text { range }\end{array}$ \\
\hline Glucose & $88 \mathrm{mg} / \mathrm{dL}$ & $70-100$ \\
\hline Sodium & $125 \mathrm{mmol} / \mathrm{L}$ & $135-148$ \\
\hline Potassium & $4.0 \mathrm{mmol} / \mathrm{L}$ & $3.5-5.3$ \\
\hline Chloride & $93 \mathrm{mmol} / \mathrm{L}$ & $92-108$ \\
\hline Bicarbonate & $18 \mathrm{mmol} / \mathrm{L}$ & $22-30$ \\
\hline Blood urea nitrogen & $17 \mathrm{mg} / \mathrm{dL}$ & $8-23$ \\
\hline Creatinine & $0.82 \mathrm{mg} / \mathrm{dL}$ & $0.50-1.00$ \\
\hline Calcium & $7.7 \mathrm{mg} / \mathrm{dL}$ & $8.2-9.6$ \\
\hline Albumin & $2.8 \mathrm{~g} / \mathrm{dL}$ & $3.8-5.1$ \\
\hline Hemoglobin ${ }^{b}$ & $9.9 \mathrm{~g} / \mathrm{dL}$ & $13.1-17.5$ \\
\hline Platelet count & $328 \times 10^{9} / \mathrm{L}$ & $150-400$ \\
\hline White blood cell count & $14.8 \times 10^{9} / \mathrm{L}$ & $4.0-10.0$ \\
\hline Hematocrit $^{b}$ & $28.4 \%$ & $40.0-51.0 \%$ \\
\hline Red blood cell count & $3.31 \times 10^{12} / \mathrm{L}$ & $4.6-6.0$ \\
\hline Mean corpuscular volume & $85.8 \mathrm{fL}$ & $80-100.0$ \\
\hline Absolute neutrophil count & $11.4 \times 10^{9} / \mathrm{L}$ & $1.7-6.5$ \\
\hline Absolute lymphocyte count & $1.3 \times 10^{9} / \mathrm{L}$ & $8.0-4.0$ \\
\hline Absolute monocyte count & $1.2 \times 10^{9} / \mathrm{L}$ & $0.2-1.0$ \\
\hline Automated absolute neutrophil count & $9.9 \times 10^{9} / L$ & $1.7-6.5$ \\
\hline Absolute eosinophil count & $1.0 \times 10^{9} / \mathrm{L}$ & $<0.6$ \\
\hline
\end{tabular}

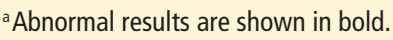

bPrevious studies showed microcytic iron deficiency anemia with low ferritin, serum iron, and transferrin saturation.

The patient returned to the subacute rehabilitation facility, where he was evaluated 2 days later. The red, purple, and maroon appearance of the skin around the right knee persisted and now involved the left knee (Figure 2). The patient was referred to a dermatologist, and results of further laboratory tests (Table 3) and skin biopsy were obtained.

2 What is the most likely cause of this pa-
tient's livedo reticularis?

Acquired thrombotic thrombocytopenic purpura

Antiphospholipid syndrome

Cholesterol embolization syndrome

Thromboangiitis obliterans 


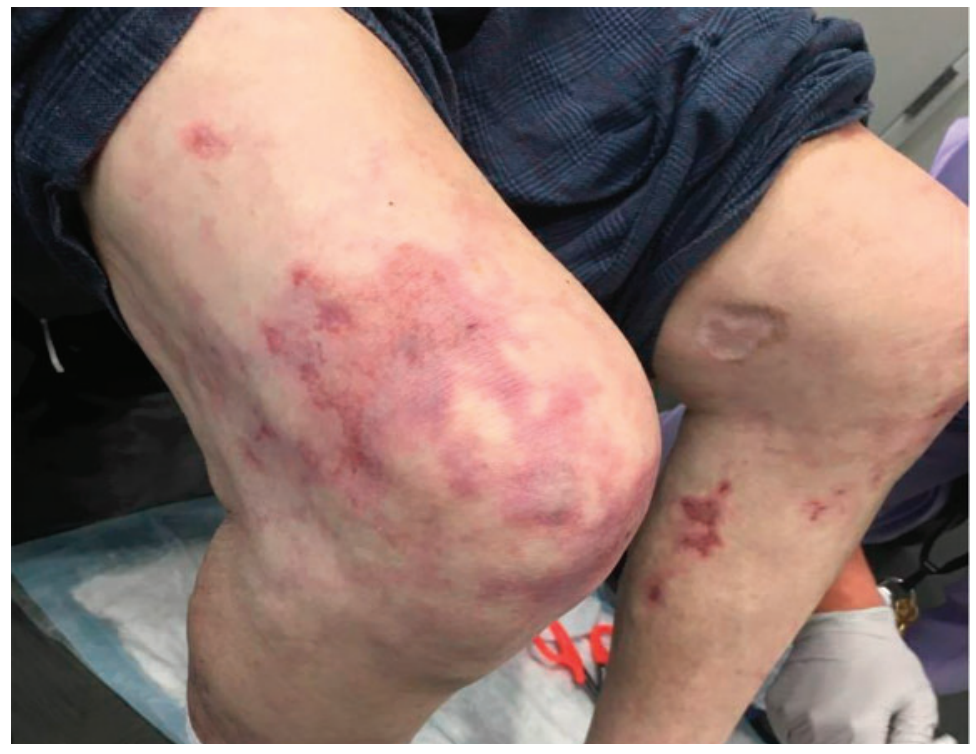

Figure 2. The patient's lower extremities demonstrated evolving livedo reticularis.

Cholesterol embolization can occur spontaneously ${ }^{9}$ but is most often reported after cardiac catheterization and infrarenal aortic and infrainguinal vascular procedures. ${ }^{10,11}$ Because our patient's skin findings developed 3 weeks after his lower extremity angiogram, choles-
Three months earlier, he underwent a left femoral endarterectomy with angioplasty terol embolization syndrome should be strongly considered in the differential diagnosis.

Cholesterol embolization syndrome starts with rupture of atherosclerotic plaque in a proximal large-caliber artery such as the aorta or iliac artery, followed by embolization of plaque debris and settling of cholesterol crystals in distal small to medium arteries. ${ }^{12}$ A foreign-body inflammatory response to the cholesterol emboli ensues. ${ }^{12,13}$ End-organ damage results from both obstruction and inflammation.

Cholesterol embolization syndrome is a microembolic process rather than what occurs in the more common arterioarterial embolization syndrome. The latter involves embolization of a large organized thrombus overlying an atheromatous plaque, which travels distally to occlude large downstream arteries and leads to severe ischemia of target organs. ${ }^{12}$

Cutaneous manifestations of cholesterol embolization syndrome include livedo reticularis with characteristic reddish-purple spots distributed in a fishnet or lacy (reticulated) pattern, cyanosis due to microvascular isch- emia, and ulceration that progresses to gangrene. ${ }^{12,14}$ The skin of the lower extremities is most commonly affected; if both extremities are involved, findings are usually asymmetric.

The diagnosis can be made clinically, with findings usually presenting days to weeks after the procedure, and with blood tests indicating inflammation, eg, elevated C-reactive protein, erythrocyte sedimentation rate, and white blood cell count with eosinophilia. ${ }^{12}$ However, because the syndrome can mimic several diseases, biopsy of an involved organ (eg, kidney, skin, muscle) is needed to confirm the diagnosis. Biopsy of a suspect skin ulcer is generally preferable to internal organ biopsy because of easy accessibility and less procedural morbidity.

Thrombotic thrombocytopenic purpura, along with other thrombotic microangiopathies, may have cutaneous manifestations, including livedo reticularis, petechiae, ecchymosis, and purpura. ${ }^{15}$ Acquired thrombotic thrombocytopenic purpura is an autoimmune disorder that results in functional inhibition or increased clearance of the ADAMTS13 enzyme, which normally cleaves von Willebrand factor to avert inappropriate platelet aggregation. It manifests as a Coombs-negative hemolytic anemia with thrombocytopenia and results in organ dysfunction, most notably in the central nervous system. The autoantibody process is often triggered by infection, drugs, malignancy, or pregnancy, although many patients do not have an identifiable cause. ${ }^{16}$

This diagnosis is unlikely in our patient. He does not have thrombosis or thrombocytopenia, his anemia is chronic, his normal total bilirubin is not concerning for hemolysis, and he does not have neurologic symptoms.

Antiphospholipid syndrome is an autoimmune disease characterized by thrombosis and, often, thrombocytopenia and red blood cell hemolysis. ${ }^{17}$ Unlike thrombotic thrombocytopenic purpura, antiphospholipid syndrome is not a microangiopathic hemolytic process, so schistocytes are not seen on peripheral smear. The pathophysiology involves inappropriate binding of antiphospholipid antibodies to endothelial surfaces, which leads to upregulation of adhesion molecules to promote inflammation and coagulation. ${ }^{18}$ Antiphospholipid syndrome often occurs in patients with other un- 
derlying autoimmune disorders (eg, systemic lupus erythematosus).

The thrombocytopenia is often mild (platelet count 50-150 × 109/L). Cutaneous manifestations commonly include livedo reticularis and livedoid vasculopathy (ie, painful and recurrent ulcerations). ${ }^{17,18}$ The lack of thrombosis, thrombocytopenia, or hemolysis makes this diagnosis unlikely in our patient.

Thromboangiitis obliterans (Buerger disease) is an inflammatory vasculitis that affects primarily small to medium-sized arteries and veins and leads to the development of occlusive thrombi. ${ }^{19}$ Although its cause remains unknown, it occurs primarily in younger men (usually before age 45) and is almost exclusively associated with tobacco use..$^{20}$

Serologic markers of inflammation including erythrocyte sedimentation rate, C-reactive protein, antinuclear antibody, rheumatoid factor, and complement levels are usually unremarkable. ${ }^{19,20}$

Although our patient is male and uses tobacco, it would be unusual to present with an initial insult at his more advanced age. Also, his peripheral arterial disease is affecting his large arteries, which is not characteristic of Buerger disease.

\section{CASE CONTINUED:} CHOLESTEROL EMBOLIZATION SYNDROME

Our patient's complete blood cell count 3 weeks after his angiogram indicated a high white blood cell count with eosinophilia. His erythrocyte sedimentation rate and C-reactive protein level were also elevated after hospitalization, although his chronically gangrenous feet likely confounded the picture.

Punch biopsy of the skin revealed embolic cholesterol crystals, confirming a diagnosis of cholesterol embolization syndrome.

3

Which of the following is true about cholesterol embolization syndrome?

Normal renal function after an event is highly unusual

It is more likely to occur after left heart catheterization than peripheral vascular procedures

It can be prevented by antiplatelet therapy before the procedure

\section{TABLE 3}

Follow-up laboratory evaluation at the time of skin biopsy

\begin{tabular}{|c|c|c|}
\hline Test & Value $^{a}$ & $\begin{array}{l}\text { Reference } \\
\text { range }\end{array}$ \\
\hline Erythrocyte sedimentation rate & $104 \mathrm{~mm} / \mathrm{hour}$ & $0-10$ \\
\hline C-reactive protein & $198.10 \mathrm{mg} / \mathrm{L}$ & $<5.00 \mathrm{mg} / \mathrm{L}$ \\
\hline $\begin{array}{l}\text { Antineutrophil cytoplasmic antibodies } \\
\text { immunoglobulin (Ig) G }\end{array}$ & $<1: 20$ & $<1: 20$ \\
\hline Rheumatoid factor & $10.7 \mathrm{IU} / \mathrm{mL}$ & $0-14.0$ \\
\hline Beta 2 glycoprotein IgG & $1 \mathrm{U} / \mathrm{mL}$ & $0-20$ \\
\hline Beta 2 glycoprotein IgM & $1 \mathrm{U} / \mathrm{mL}$ & $0-20$ \\
\hline Cardiolipin IgA & 9.9 APL units & $0.0-13.9$ \\
\hline Cardiolipin IgG & 6.7 GPL units & $0-9.9$ \\
\hline Cardiolipin IgM & $<2.5 \mathrm{MPL}$ units & $0-9.9$ \\
\hline Nuclear antibody lgG & $<1: 40$ & $<1: 40$ \\
\hline
\end{tabular}

${ }^{a}$ Abnormal results are shown in bold.

Subsequent angioplasty is not contraindicated

It is unknown if cholesterol embolization syndrome is a risk factor for recurrence after a subsequent similar procedure. ${ }^{12,21}$ Hence, angioplasty procedures after diagnosis are not absolutely contraindicated, but careful risk-benefit analysis should be done since the initial occurrence suggests the presence of atherosclerotic plaque that may still be vulnerable to mechanical injury and repeat embolization. Whether benefits of further interventions likely outweigh the potential risk of recurrent cholesterol emboli should be considered on an individual basis.

About half of patients with cholesterol embolization syndrome have kidney involvement, presenting as a rise in serum creatinine with proteinuria. One mechanism of renal injury is thought to be vascular obstruction by cholesterol crystals, leading to tissue ischemia, cell necrosis, and inflammation.

The gastrointestinal tract is affected in $19 \%$ to $48 \%$ of patients, and the problem most commonly presents as bowel ischemia, which can result in gastrointestinal blood loss from mucosal ulcerations due to mucosal infarcts.

Cutaneous manifestations occur in 35\% to

\section{Cholesterol embolization can occur spontaneously, but most often after vascular procedures}




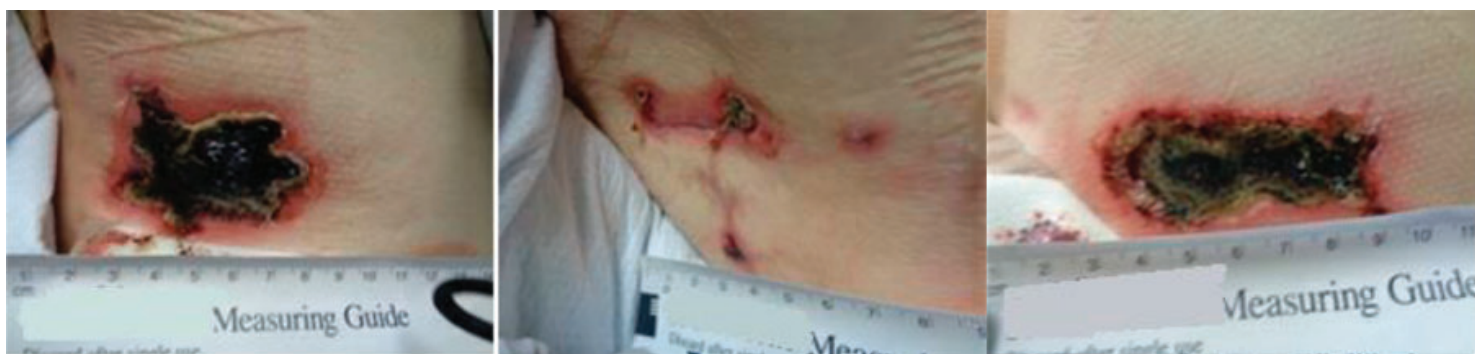

Figure 3. The patient's right flank (left), lower back (middle), and right hip (right) demonstrated evolving ulceration and necrosis of lesions seen in Figure 1.

$96 \%$ of patients, and those with renal manifestations are most commonly affected..$^{12,22}$ Although a decrease in renal function would support a diagnosis of cholesterol embolization syndrome, it is not uncommon for kidneys to be unaffected, as is the case for our patient.

Based on observational data, patients undergoing cardiac surgery or abdominal aortic repair are at more risk of developing cholesterol embolization syndrome than those having coronary angiography or minimally invasive procedures. ${ }^{12}$ Its incidence after angiography or angioplasty is low $(1 \%-2 \%)$, but it is likely underrecognized. ${ }^{23,24}$

Although cholesterol embolization syn-

Punch biopsy

of the skin

revealed

embolic

cholesterol

crystals,

confirming

the diagnosis drome can develop after angiography alone as a result of catheter introduction or manipulation, interventional procedures may increase the risk. A high index of suspicion for cholesterol embolization syndrome should be maintained regardless of the indication for angiography, arterial access site, or interventions performed.

No clinical evidence suggests that antiplatelet therapy helps prevent cholesterol embolization syndrome. Modifiable risk factor reduction (eg, blood pressure control, smoking cessation) may help prevent it. ${ }^{23}$

\section{SUPPORTIVE MANAGEMENT}

No specific treatments address cholesterol embolization syndrome, so management is primarily supportive by maintaining hemodynamic stability and optimizing nutrition. Endorgan damage is associated with high morbidity and mortality risk and is particularly important to address. ${ }^{12}$

Severe skin lesions require appropriate wound care, appropriate bed support surfaces, and patient positioning to protect from fur- ther injury and secondary infection. ${ }^{25}$

Steroids have been suggested to reduce inflammation caused by cholesterol crystals, ${ }^{26,27}$ as have statins to help stabilize plaques. ${ }^{28,29}$ However, neither therapy has been studied for cholesterol embolization syndrome in a randomized controlled trial.

Recurrent embolism rates have been reduced with surgical endarterectomy and bypass procedures, and data on the use of intraluminal stenting procedures is promising in some patients. ${ }^{30}$ There is no role for anticoagulation or fibrinolytic therapy for managing the condition.

Some case series suggest that warfarin or fibrinolytics may lead to cholesterol embolization syndrome, although this association is unclear and not supported by clinical trial data. The best way to reduce recurrence may be to limit invasive intra-arterial studies and interventions. ${ }^{12}$ Unfortunately, this is challenging, as many of these patients require such studies and procedures, given their medical complexity and severity of cardiovascular disease.

\section{CASE CONTINUED: ULCER CARE}

The patient had normal renal function, indicating his kidneys had been spared. He required no treatment other than care of the skin ulcers that developed from damage by cholesterol emboli.

Over the course of 4 weeks, his lesions, initially confluent, reticulated, and purplish, evolved into full-thickness necrotic ulcers (Figure 3). Four demarcated ulcers on his back and trunk (the largest being $3.5 \mathrm{~cm} \times 8$ $\mathrm{cm}$ ) were $100 \%$ necrotic (eschar) tissue with open wound edges that drained a moderate amount of serosanguinous fluid.

Initially, a dry sodium-impregnated gauze 
was used to debride the moist necrotic tissue through reverse osmosis. As the necrotic areas softened and were debrided, the wound base developed granulation buds with areas of thick, slough tissue (Figure 4). The wound was then managed with a honey-based product to address the high bacterial load in the ulcers, followed by a calcium alginate foam dressing to absorb the exudate while maintaining a moist wound. Surgical debridement was not required, and neither systemic nor topical antibacterial agents were used.

Five months after the patient's hospitalization, the ulcers had improved, and hydrocolloid dressings were used. At 8 months, all the ulcers were healed.

Unfortunately, his severe gangrene of the toes, which predated the cholesterol embolization syndrome, progressed to involve the midfoot and eventually required bilateral above-the-knee amputation. Once the patient was less catabolic from chronic gangrene, and after receiving excellent wound care and optimization of oral nutrition, the ulcers from his cholesterol emboli healed completely, and he regained his previous baseline health status.

\section{TAKE-AWAY POINTS}

- Skin lesions involving the disruption of arteries and subsequent ecchymosis, whatever the inciting mechanism, should prompt an evaluation for an underlying systemic cause.

- Cholesterol emboli should be suspected when characteristic skin lesions appear in

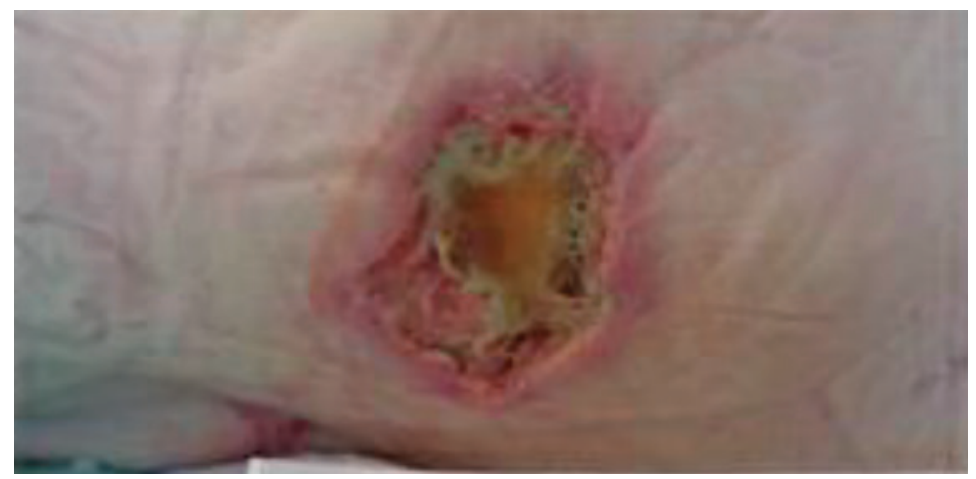

Figure 4. The patient's flank ulcer 2 months after treatment.

the days or weeks after angiography.

- Clinical history, physical examination, laboratory findings, and biopsy are essential for diagnosis.

- Renal and gastrointestinal complications are potentially the most serious end-organ manifestations of cholesterol embolization syndrome. However, skin lesions can progress to large necrotic ulcers, causing significant morbidity.

- No treatment is specific for cholesterol embolization syndrome, but complications must be managed.

- Smoking cessation and blood pressure control may reduce the risk.

- Substantial damage to skin can occur in cholesterol embolization syndrome, as in this case, with 8 months to complete resolution of what eventually became fullthickness necrotic ulcers.

Acknowledgment: The authors would like to thank Sheila Howes, MSN, APRN, FNP-BC, CWON, CFCN, for her assistance with the technical description of the wound care.

\section{REFERENCES}

1. Sajjan VV, Lunge S, Swamy MB, Pandit AM. Livedo reticularis: a review of the literature. Indian Dermatol Online J 2015; 6(5):315-321. doi:10.4103/2229-5178.164493

2. Gibbs MB, English JC 3rd, Zirwas MJ. Livedo reticularis: an update. J Am Acad Dermatol 2005; 52(6):1009-1019. doi:10.1016/j.jaad.2004.11.051

3. Edsberg LE, Black JM, Goldberg M, McNichol L, Moore L, Sieggreen M. Revised National Pressure Ulcer Advisory Panel Pressure Injury Staging System: revised pressure injury staging system. J Wound Ostomy Continence Nurs 2016; 43(6):585-597. doi:10.1097/WON.0000000000000281

4. Bhattacharya S, Mishra RK. Pressure ulcers: current understanding and newer modalities of treatment. Indian J Plast Surg 2015; 48(1):4-16. doi:10.4103/0970-0358.155260

5. Mookadam F, Cikes M. Images in clinical medicine. Cullen's and Turner's signs. N Engl J Med 2005; 353(13):1386. doi:10.1056/NEJMicm040796

6. Carnevale-Maffé G, Modesti PA. Out of the blue: the Grey-Turner's sign. Intern Emerg Med 2015; 10(3):387-388. doi:10.1007/s11739-014-1178-0

7. Talwar A, Kumar S, Gopal MG, Nandini AS. Spectrum of purpura fulminans: report of three classical prototypes and review of management strategies. Indian J Dermatol Venereol Leprol 2012; 78(2):228. doi:10.4103/0378-6323.93655

8. Thornsberry LA, LoSicco KI, English JC 3rd. The skin and hypercoagulable states. J Am Acad Dermatol 2013; 69(3):450-462. doi:10.1016/j.jaad.2013.01.043

9. Carmona Sánchez P, González Serrano MT, Serrano Simón JM. Spontaneous multivisceral cholesterol crystal embolism presenting as livedo reticularis. Med Intensiva 2018; 42(4):e4. doi:10.1016/j.medin.2016.08.006

10. Sharma PV, Babu SC, Shah PM, Nassoura ZE. Changing patterns of atheroembolism. Cardiovasc Surg 1996; 4(5):573-579. doi:10.1016/0967-2109(96)00003-8

11. Quinones A, Saric M. The cholesterol emboli syndrome in atherosclerosis. Curr Atheroscler Rep 2013; 15(4):315. doi:10.1007/s11883-013-0315-y

12. Kronzon I, Saric M. Cholesterol embolization syndrome. Circulation 
2010; 122(6):631-641. doi:10.1161/CIRCULATIONAHA.109.886465

13. Samstad EO, Niyonzima N, Nymo S, et al. Cholesterol crystals induce complement-dependent inflammasome activation and cytokine release. J Immunol 2014; 192(6):2837-2845. doi:10.4049/jimmunol.1302484

14. Donohue KG, Saap L, Falanga V. Cholesterol crystal embolization: an atherosclerotic disease with frequent and varied cutaneous manifestations. J Eur Acad Dermatol Venereol 2003; 17(5):504-511. doi:10.1046/j.1468-3083.2003.00710.x

15. Kappler S, Ronan-Bentle S, Graham A. Thrombotic microangiopathies (TTP, HUS, HELLP). Hematol Oncol Clin North Am 2017; 31(6):1081-1103. doi:10.1016/j.hoc.2017.08.010

16. Knöbl P. Thrombotic thrombocytopenic purpura. Memo 2018; 11(3):220-226. doi:10.1007/s12254-018-0429-6

17. Garcia D, Erkan D. Diagnosis and management of the antiphospholipid syndrome. N Engl J Med 2018; 378(21):2010-2021. doi:10.1056/NEJMra1705454

18. Giannakopoulos B, Krilis SA. The pathogenesis of the antiphospholipid syndrome. N Engl J Med 2013; 368(11):1033-1044. doi:10.1056/NEJMra1112830

19. Olin JW, Shih A. Thromboangiitis obliterans (Buerger's disease). Curr Opin Rheumatol 2006; 18(1):18-24. doi:10.1097/01.bor.0000198000.58073.aa

20. Olin JW. Thromboangiitis obliterans (Buerger's disease). N Engl J Med 2000; 343(12):864-869. doi:10.1056/NEJM200009213431207

21. Palmer FJ, Warren BA. Multiple cholesterol emboli syndrome complicating angiographic techniques. Clin Radiol 1988; 39(5):519-522. doi:10.1016/s0009-9260(88)80221-6

22. Li X, Bayliss G, Zhuang S. Cholesterol crystal embolism and chronic kidney disease. Int J Mol Sci 2017; 18(6):1120. doi:10.3390/ijms18061120

23. Fukumoto Y, Tsutsui H, Tsuchihashi M, Masumoto A, Takeshita A;
Cholesterol Embolism Study(CHEST) Investigators. The incidence and risk factors of cholesterol embolization syndrome, a complication of cardiac catheterization: a prospective study. J Am Coll Cardiol 2003; 42(2):211-216. doi:10.1016/s0735-1097(03)00579-5

24. Agrawal A, Ziccardi MR, Witzke C, Palacios I, Rangaswami J. Cholesterol embolization syndrome: an under-recognized entity in cardiovascular interventions. J Interv Cardiol 2018; 31(3):407-415. doi:10.1111/joic.12483

25. Belenfant X, Meyrier A, Jacquot C. Supportive treatment improves survival in multivisceral cholesterol crystal embolism. Am J Kidney Dis 1999; 33(5):840-850. doi:10.1016/s0272-6386(99)70415-4

26. Fabbian F, Catalano C, Lambertini D, Bordin V, Di Landro D. A possible role of corticosteroids in cholesterol crystal embolization. Nephron 1999; 83(2):189-190. doi:10.1159/000045509

27. Nakayama M, Nagata M, Hirano $T$, et al. Low-dose prednisolone ameliorates acute renal failure caused by cholesterol crystal embolism. Clin Nephrol 2006; 66(4):232-239. doi:10.5414/cnp66232

28. Abela GS, Vedre A, Janoudi A, Huang R, Durga S, Tamhane U. Effect of statins on cholesterol crystallization and atherosclerotic plaque stabilization. Am J Cardiol 2011; 107(12):1710-1717. doi:10.1016/j.amjcard.2011.02.336

29. Woolfson RG, Lachmann H. Improvement in renal cholesterol emboli syndrome after simvastatin. Lancet 1998; 351(9112):1331-1332. doi:10.1016/S0140-6736(05)79058-9

30. Shames ML, Rubin BG, Sanchez LA, Thompson RW, Sicard GA. Treatment of embolizing arterial lesions with endoluminally placed stent grafts. Ann Vasc Surg 2002; 16(5):608-612. doi:10.1007/s10016-001-0278-2

Address: Roberta Meyers, MD, MPH, Department of Medicine, Hennepin Healthcare System, 701 Park Avenue S, Minneapolis, MN 55415;

roberta.meyers@hcmed.or 